\title{
Three-Dimensional Reconstruction with a Laser Line Based on Image In-Painting and Multi-Spectral Photometric Stereo ${ }^{\dagger}$
}

\author{
Liang Lu ${ }^{1}$, Hongbao Zhu ${ }^{1}$, Junyu Dong ${ }^{1, *}$, Yakun Ju ${ }^{1}$ and Huiyu Zhou ${ }^{2}$ \\ 1 College of Information Science and Engineering, Ocean University of China, Qingdao 266100, China; \\ luliang@stu.ouc.edu.cn (L.L.); zhuhongbao@stu.ouc.edu.cn (H.Z.); juyakun@stu.ouc.edu.cn (Y.J.) \\ 2 School of Informatics, University of Leicester, Leicester LE1 7RH, UK; hz143@leicester.ac.uk \\ * Correspondence: dongjunyu@ouc.edu.cn \\ + This article is an extended version of our prior work "Image in-painting of multi-spectral image with laser \\ lines based on Generative Adversarial Network", which was accepted by the fifth International Conference on \\ Machine Vision and Information Technology (CMVIT 2021), which was held on 26 February 2021, virtually.
}

check for updates

Citation: Lu, L.; Zhu, H.; Dong, J.; Ju, Y.; Zhou, H. Three-Dimensional Reconstruction with a Laser Line Based on Image In-Painting and Multi-Spectral Photometric Stereo . Sensors 2021, 21, 2131. https:// doi.org/10.3390/s21062131

Academic Editor: Chih-Hsien Hsia

Received: 10 February 2021

Accepted: 7 March 2021

Published: 18 March 2021

Publisher's Note: MDPI stays neutral with regard to jurisdictional claims in published maps and institutional affiliations.

Copyright: (c) 2021 by the authors. Licensee MDPI, Basel, Switzerland. This article is an open access article distributed under the terms and conditions of the Creative Commons Attribution (CC BY) license (https:/ / creativecommons.org/licenses/by/ $4.0 /)$.

\begin{abstract}
This paper presents a multi-spectral photometric stereo (MPS) method based on image in-painting, which can reconstruct the shape using a multi-spectral image with a laser line. One of the difficulties in multi-spectral photometric stereo is to extract the laser line because the required illumination for MPS, e.g., red, green, and blue light, may pollute the laser color. Unlike previous methods, through the improvement of the network proposed by Isola, a Generative Adversarial Network based on image in-painting was proposed, to separate a multi-spectral image with a laser line into a clean laser image and an uncorrupted multi-spectral image without the laser line. Then these results were substituted into the method proposed by Fan to obtain high-precision 3D reconstruction results. To make the proposed method applicable to real-world objects, a rendered image dataset obtained using the rendering models in ShapeNet has been used for training the network. Evaluation using the rendered images and real-world images shows the superiority of the proposed approach over several previous methods.
\end{abstract}

Keywords: image in-painting; generative adversarial network; multi-spectral photometric stereo; and laser extraction

\section{Introduction}

Three-dimensional reconstruction has been one of the active research areas in computer vision for several decades. There have been many algorithms that can perform high-precision 3D reconstruction of the target, including laser scanning technology [1-3], photometric stereo [4], structure from motion [5], multi-view stereo [6], etc. There is a recent trend that attempts to combine deep learning with geometry-based 3D reconstruction methods e.g., photometric stereo. However, many issues exist when such 3D reconstruction methods are used in an uncontrolled environment, e.g., underwater 3D imaging: (1) Image acquisition is difficult, while deep learning usually requires a large amount of data for training; (2) The images required by photometric stereo algorithms should be obtained when the camera and the target are relatively still, while it is difficult to achieve in an underwater environment; (3) The result obtained by the photometric stereo algorithm does not have scale accuracy, that is, only the shape is relatively accurate, and the height information is not accurate.

In order to solve the first issue, we can use the rendered images as the training set [7]. For the second issue, we could use a multi-spectral photometric stereo algorithm [8-10], which only needs a single-color image [11-14]. Finally, for the third issue, we could add a laser line on the RGB image, and correct the result of the multi-spectral photometric stereo algorithm through the height information of the pixels on the laser line $[15,16]$.

However, in the process of 3D reconstruction using multi-spectral images with laser lines, there are the following problems: (1) The laser line will pollute the imaging result 
of the color light source, that is, the area covered by the laser light source will lose pixel information; (2) The color light source will influence the result of laser line extraction.

Inspired by the strong ability of Generation Adversarial Networks (GAN) to model the data distribution, in this study, we use GAN based on image in-painting to solve the above issues. Through the improvement of the network proposed by Isola [17], we proposed a network which can separate the multi-spectral image with a laser line into a clean laser image and an uncorrupted multi-spectral image without the laser line. And based on the proposed network and Fan's [16] algorithm, we achieved accurate 3D reconstruction using a multispectral image with a laser line.

The overview of the proposed method is shown in Figure 1.

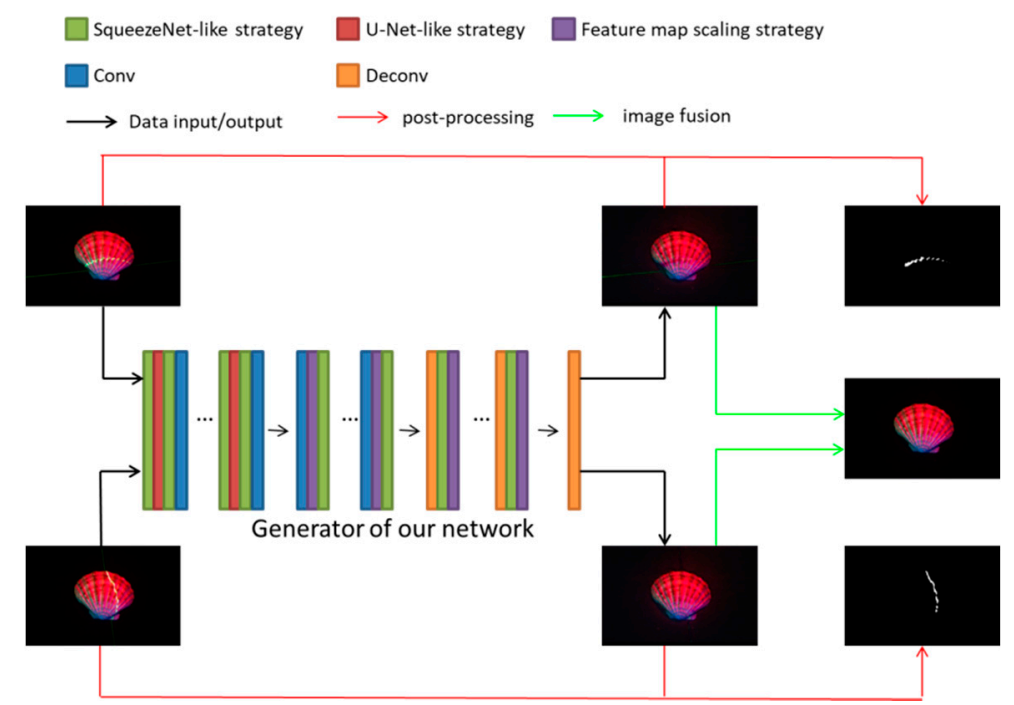

Figure 1. The overview of the proposed method. Input images are illuminated with red, green and blue lights simultaneously together with a projected laser line. We propose a network based on Generation Adversarial Networks (GAN) and image in-painting to separate the multi-spectral image with a laser line into a clean laser image and an uncorrupted multi-spectral image without the laser line. The details of the three strategies represented by green, red and purple will be introduced in Section 3.1, and the post-processing represented by the red and green arrows will be introduced in Section 4.2.

The main contributions of this article are summarized as follows:

(1) Through the improvement of the network proposed by Isola [17], we propose a Generative Adversarial Network based on image in-painting to realize the effective estimation of the pixel values at the locations covered by the laser line in the multispectral image;

(2) The proposed network can effectively extract the laser line in the multi-spectral image;

(3) Through adding certain U-Net-like structures to the generator of GAN, the proposed network can produce stable results;

(4) Based on the proposed network and Fan's [16] algorithm, we achieve accurate 3D reconstruction using a multispectral image with a laser line.

The remaining of this article is organized as follows: Section 2 introduces the related work; Section 3 introduces the network structure, related parameters and training process of the Generative Adversarial Network proposed in this article; Section 4 introduces the rendered image dataset used in this article and the results of the rendered images and the real images; Section 5 concludes this paper.

\section{Related Work}

In this section, we firstly make an introduce on the traditional multi-spectral photometric stereo in Section 2.1, and then make an introduce on photometric stereo algorithm 
based on laser line correction in Section 2.2, finally, we briefly review some traditional methods on laser line extraction algorithms in Section 2.3, and some image in-painting algorithms based on deep learning in Section 2.4.

\subsection{Introduction of Multi-Spectral Photometric Stereo}

The traditional multi-spectral photometric stereo technique can reconstruct the 3D geometry with a color image. The image should be obtained under the trichromatic light source with known angles.

The principle of multi-spectral photometric stereo is shown in Equation (1).

$$
c_{i}(\mathrm{x}, \mathrm{y})=\sum_{i} l_{j}^{T} n(x, y) \int E_{j}(\lambda) R(x, y, \lambda) S_{i}(\lambda) d \lambda
$$

where, $l_{j}$ is the $j$-th illumination direction vector, $n(x, y)$ is the normal vector of a certain point of the target, $E_{j}(\lambda)$ is the illumination intensity, $R(x, y, \lambda)$ is a parameter related with the albedo and chromaticity of a certain point of the target, and $S_{i}(\lambda)$ is the color response of the camera photosensitive element.

Assume $R(x, y, \lambda)$ as the product of $\rho(x, y)$ and $\alpha(\lambda)$, which represent the albedo and the chromaticity respectively, then combining all the terms which are related with $\lambda$ as a whole, and we can get a parameter matrix $V$, as shown in Equation (2).

$$
V_{i j}=\int E_{j}(\lambda) \alpha(\lambda) S_{i}(\lambda) d \lambda
$$

So we can rewrite Equation (1) as Equation (3), and obtain Equation (4).

$$
\begin{gathered}
C=V L \rho n \\
n=\frac{V^{-1} L^{-1} c}{\left\|V^{-1} L^{-1} c\right\|}
\end{gathered}
$$

That is, the exact solution of the normal vector of the target surface can be obtained on the premise that the target's chromaticity and the illumination direction are known.

However, these formulas are based on images acquiring in an ideal state. Since the chromaticity of each point on the surface of the target are unpredictable, and meanwhile, the near-field light source, instead of the parallel light source, is used in practical applications, which is assumed in the traditional multi-spectral photometric stereo algorithm, the direction and the intensity of the light sources are also different for each point on the surface of the target. That is, in formula (2), the parameter matrix $V_{i j}$ of each piont are different cause the $E_{j}(\lambda)$ and the $\alpha(\lambda)$ of each piont are different, and in formula (4), the $L$ of each point are different. Therefore, it is impossible to obtain high-precision reconstruction results only by applying traditional multi-spectral photometric stereo algorithms.

\subsection{Introduction of Photometric Stereo Algorithm Based on Laser Line Correction}

Fan [16] proposed an algorithm, which could obtain the high-precision reconstruction results, through correcting the $3 \mathrm{D}$ reconstruction results obtained by the photometric stereo algorithm by laser triangulation. The overview of the Fan's algorithm is shown in Figure 2.

The reconstruction result of the FAN's algorithm can achieve high accuracy, but it is required that the camera and the target remain relatively still during the image acquisition process. The motivation of this manuscript is to use deep learning to decompose a multispectral image with laser lines into a multi-spectral image without laser lines, and a pure laser line image, and at first calculate the rough 3D shape through the traditional multispectral photometric stereo algorithm, then, obtain the height information of the area covered by the laser line through the laser triangulation algorithm, and finally, correct the reconstruction result of the target surface through the algorithm proposed by Fan. 

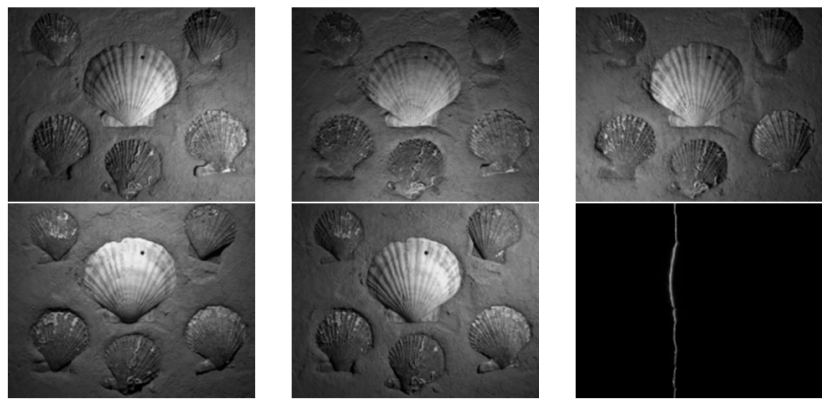

(a) Input images
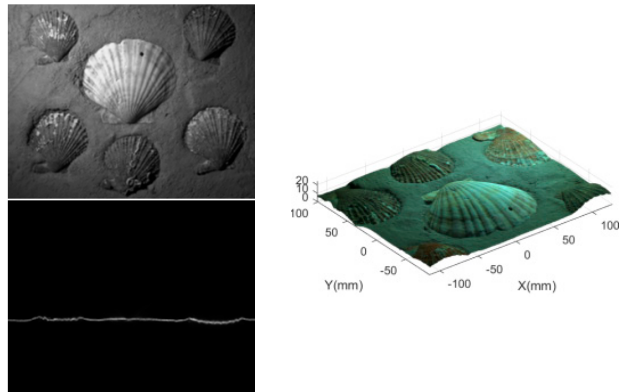

(b) Result

Figure 2. The overview of the Fan's algorithm. (a) are the 8 input images, including 6 gray images which are acquired on 6 different angles, and 2 laser images acquired when the laser source is the only illumination source, respectively, (b) is the reconstruction result.

\subsection{Laser Line Extraction Algorithms}

The laser scanning method is one of the earliest proposed and fully studied 3D reconstruction methods, and even commercially available [18], but most of these devices or algorithms can only process the laser line extraction problem when the laser is the only light source or the ambient light is very weak relative to the laser brightness. In terms of algorithmic development in this field, there are many kinds of research studies on laser line thinning. Molder et al. [19] proposed and studied two laser line center position detection methods, which improved the laser line detection results to sub-pixel accuracy. Natalija et al. [20] proposed a new laser line center estimation algorithm. Li et al. [21] proposed the gray gravity method to extract the center of laser stripes. Ta et al. [22] proposed a laser line detection method, using the color space to enhance the laser signal and reduce the discrimination effect of white ambient light, to improve the accuracy of the line detection algorithm. Song et al. [23] proposed a hybrid laser image point extraction algorithm using SVD decomposition in the HSV space of the image. Pavel et al. [24] proposed a laser line extraction algorithm based on color segmentation.

However, the premise of the above algorithm for laser line detection is that the intensity of the background and the laser line is significantly different, such as natural light [22,23], or dark illumination environments [24], which is not applicable to the extraction of laser lines in trichromatic laser images.

\subsection{Image In-Painting Algorithms Based on Deep Learning}

Over the last few years, deep learning techniques have yielded significant improvements in image in-painting. Liu et al. [25] proposed a novel coherent semantic attention (CSA) layer to construct the correlation between the deep features of hole regions, and introduced a consistency loss to guide the CSA layer to learn the VGG features of ground truth, to achieve better in-painting results. Hong et al. [26] designed a learnable fusion block, which predicts an alpha composition map to achieve smooth transition, to implement pixel level fusion in the transition region. Yu et al. [27] proposed a unified feed-forward generative network with a novel contextual attention layer for image in-painting. Then they [28] proposed a generative image in-painting system based on gated convolutions to complete images with free-form mask and guidance. Nazeri et al. [29] proposed a two-stage adversarial model EdgeConnect which comprises of an edge generator and an image completion network. The network can hallucinate edges of the missing region through the edge generator and fill in the missing regions through the image completion network. Zeng et al. [30] proposed an iterative in-painting method using a deep generative model with a feedback mechanism, and a guided up-sampling network to enable generation of high-resolution in-painting results. Huang et al. [31] proposed a method for automatically guiding patch-based image completion using mid-level structural cues. Yang et al. [32] proposed a novel pyramid structure loss is proposed to supervise structure learning and embedding, and an attention mechanism is developed to further exploit the re- 
current structures and patterns in the image to refine the generated structures and contents. Liu et al. [33] proposed a mutual encoder-decoder network for image in-painting, and a feature equalization method to make structure and texture features consistent with each other, to remove blur and artifacts caused by inconsistent structure and texture features. Jimmy et al. [34] proposed a novel CNN architecture, which can efficiently equip conventional CNN with the ability to learn translation variant operations for irregularly spaced data, and obtain superior performance on both image in-painting and super-resolution. Li et al. [35] proposed a Recurrent Feature Reasoning (RFR) module, which exploits the correlation between adjacent pixels and strengthens the constraints for estimating deeper pixels, leading to better results with exquisite details.

Although image in-painting algorithms based on deep learning have been greatly developed, these algorithms cannot directly deal with the problem in this article. Cause they are either suitable for blind image in-painting $[27,28]$, or for specific images (such as face images) and images under natural light. There is not an image in-painting algorithm for multispectral images. In addition, most of these existing algorithms need to provide a mask map, or calculate the mask map through interactive operations, but in this article, the laser image (i.e., the mask map) is unknown.

\section{Method}

In this section, the details of the algorithm we proposed will be introduced, which mainly include the architecture of the network, the parameter setting and training information of the Generative Adversarial Network we proposed. We first present the architecture of our network in Section 3.1, and detail the parameter settings, loss function and training process in Section 3.2.

\subsection{Architecture}

We designed an improved Generative Adversarial Network base on the network proposed by Isola [17]. The architecture of the proposed network contains twenty-one layers with different weights, where the generator part is composed of an encoder-decoder network composed of eight encoders and eight decoders, and the discriminator is composed of five convolutional networks. We did not make any changes to the discriminant network, but only improved the generator network.

The architecture of the proposed network is shown in Figure 3, and the details of our generator are expounded in Table 1.

The network proposed by Isola is mainly suitable for tasks such as synthesizing photos from label maps, reconstructing objects from edge maps, and coloring images. That is, the original network will operate on all pixels in the image. In the problem that this manuscript needs to deal with, we hope the network can reduce the operation of pixels in other locations as much as possible while operating the pixels in the area covered by the laser line. Therefore, we have introduced several strategies to realize the attention mechanism so that our proposed network can autonomously determine the location of the area covered by the laser line.

A U-Net-like strategy and a SqueezeNet-like strategy were adopted in the first few layers of the generator to obtain more complete image features, and a scaling strategy of feature map was applied in the other layers of generator to acquire more stable outputs.

U-Net-like strategy The U-Net-like strategy of our network contains four convolutional layers and three de-convolutional layers, with the parameter "striders" set to 2 . So the scale of the output of the network is reduced to one-half relative to its input, that is, we use it to replace a convolution strategy with the parameter "strider" is 2 , or a simple pooling strategy. 


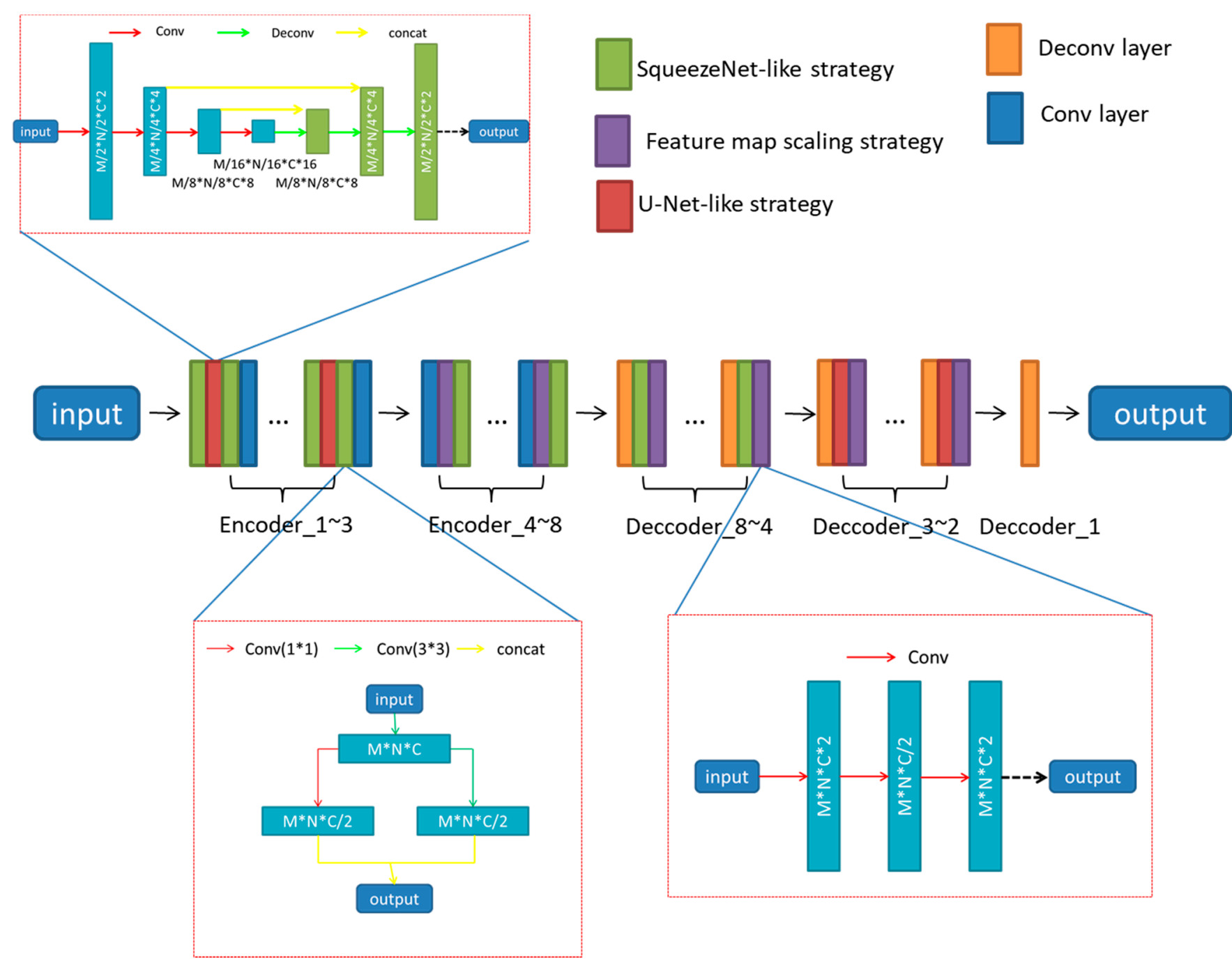

Figure 3. The detailed structure of the generator. M, N, and C are the width, height and channel of the feature map of its input value, respectively.

Table 1. Details of our proposed network.

\begin{tabular}{ccc}
\hline Name & Input_Channels & Output_Channels \\
\hline Encoder_1 & 3 & 64 \\
Encoder_2 & 64 & 128 \\
Encoder_3 & 128 & 256 \\
Encoder_4 & 256 & 512 \\
Encoder_5 8 & 512 & 512 \\
Decoder_8 5 & 512 & 512 \\
Decoder_4 & 512 & 256 \\
Decoder_3 & 256 & 128 \\
Decoder_2 & 128 & 64 \\
Decoder_1 & 64 & 3 \\
\hline
\end{tabular}

SqueezeNet-like strategy The SqueezeNet-like strategy was used to get more finegrained features. The strategy contains two convolutional layers, and a concatenation layer, the parameters "kernel_size" of the convolutional layer was set to $1 * 1$ and $3 * 3$, respectively.

Feature map scaling strategy The Feature map scaling strategy was used to acquire more stable outputs. The strategy contains three convolutional layers.

Novelty As far as we know, no other methods have been found to reduce the scale of feature maps through the U-NET-like strategy, and either the SqueezeNet-like strategy 
or the strategy which up/down the number of feature map are usually used to classify images. We are the first to use these strategies to solve our problem, and the experiment results prove the effectiveness of our improvement. The verification experiment results are shown in Section 4.3.2.

The improved generator network contains a total of 135 convolution or deconvolution layers. Among them, Encoder 1 to Encoder 3 have 14 layers respectively, including two SqueezeNet-like strategies and one U-NET-like strategy, and a convolutional layer; Encoder 4 to Encoder 8 have 7 layers respectively, Including a SqueezeNet-like strategy, a feature map scaling strategy, and a convolutional layer; decoder 8 to decoder 4 , each has 7 layers, including a deconvolution layer, a SqueezeNet-like strategy, and a feature map scaling Strategy, decoder 3 to decoder 2, each has 11 layers, including a deconvolution layer, a U-NET-like strategy, and a feature map scaling strategy. Decoder 1 has only one deconvolution layer.

The input values of all layers should be activated before performing other operations, the activate function for all the generator layers is the Leaky ReLU non-linearity function with the negative slope 0.2 , while the activate function of de-convolution layers is ReLU. In decoder_8 to decoder_3, a dropout strategy was adopted with the parameter 'keep_prob' set to 0.5 . All of these outputs value of the coder-encoder layers in the generator should be batch normalized before output. And in the final layer of decoder, an activation function 'tanh' was adopted.

\subsection{Training}

The lack of data makes difficult to train the network with real objects. Inspired by recent advances that use synthetic data for training the deep networks, we also train our network with synthetic images rendered using the ShapeNet dataset. The dataset contains 55 common object categories with about 51,300 unique 3D models. We render the 3D models based on a script on GitHub (https:/ / github.com/panmari/stanford-shapenet-renderer, accessed on 27 November 2017), which can render models to 2D images at different angles with Blender. We have improved the script so that it can generate 2D images at different angles of view for the same target illuminated by the red, green and blue light sources and a laser source.

The loss function was based on conventional 12 residual minimization and that with 11 residual minimization, and their sum was optimized using the Adam Optimizer [36]. We initialize the weights with a zero-mean Gaussian distribution and a standard deviation of 0.02 , and the learning rate is set to 0.0005 .

\section{Experiments}

In this section, we will evaluate the experimental results of the proposed method, including both rendered and real-world images. All of these results were acquired with a TESLA K40C graphics card.

\subsection{Dataset}

\subsubsection{Rendered Image Dataset}

We use the Blender Python API to render a total of 13,977 models of five categories in ShapeNet, such as bus, car, airplane, ship and train, into multispectral images with laser lines, multispectral images without laser lines, and laser images.

The settings were as follows: the resolution of the rendered images was set to 256 * 256 ; the origin of the coordinate system was set to the center of these models; the lamp type was set to "spotlight", and their beam were set to 60 degrees, the initial position were set to be at 0 degrees, 120 degrees and 240-degree, the optical axis points to the origin and can move along the ring as a whole at 15 degrees per time; 501 red spotlights were set to simulating the effect of a laser illumination, while their beam was set to 1 degree, the position moved along a straight line, and the color was set to $(1,0,0)$ or $(0,1,0)$, representing red or green laser, respectively. Each model would be rendered to 24 multi-spectral images without 
laser lines, 24 multi-spectral images with laser lines, and one laser image. Partly of these rendered images are shown in Figure 4.
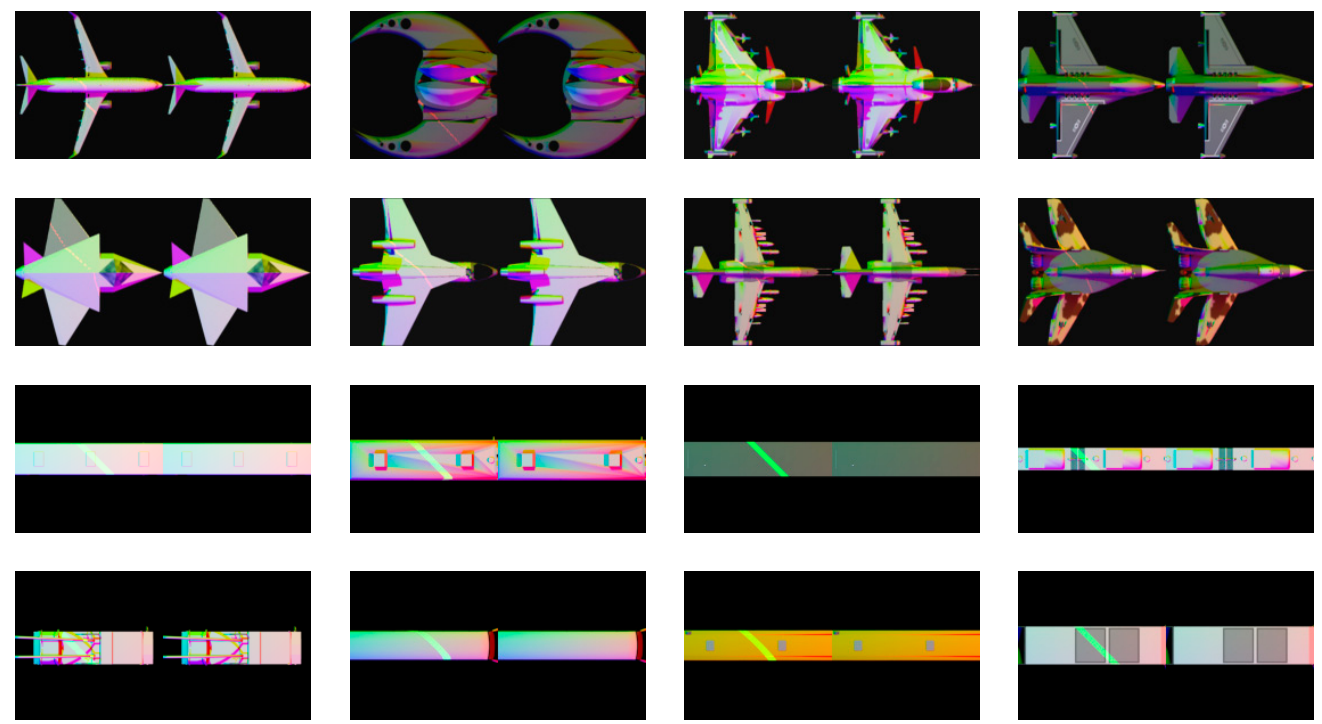

Figure 4. Partly of these rendered images. The left side of each image is a multi-spectral image with laser lines, and the right is a multi-spectral image without laser lines.

\subsubsection{Real-World Images}

We use four objects, including the shell, the ship model, the airplane model and the train model, to create a collection of real-world images at different angles, including multi-spectral images with laser lines, multi-spectral images without laser lines and the pure laser images.

Partly of these real-world images are shown in Figure 5.
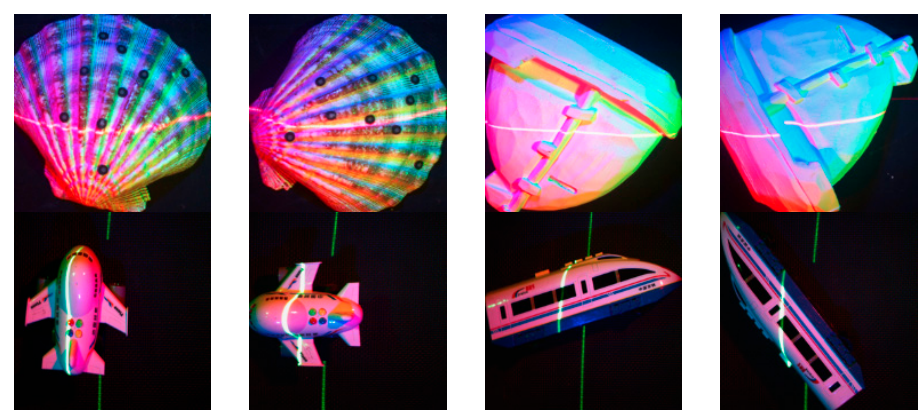

Figure 5. Partly of these real-world images. The images above are multi-spectral images with a red laser line, and the images below are multi-spectral images with a green laser line.

\subsection{Laser Line Extraction Results}

We input the multi-spectral image with a laser line into the proposed GAN network to obtain an intermediate result image. After making a difference between it and the input image, we then obtain the desired laser image through threshold segmentation, median filtering, dilation and corrosion. The threshold is set to 0.35 in the experiment.

\subsubsection{Extraction Results of Laser Lines in Rendered Images}

First, we input the rendered image into our proposed network for training 100 times, which takes about $5 \mathrm{~h}$ with a TESLA K40C graphics card. The training process is shown in Figure 6. 


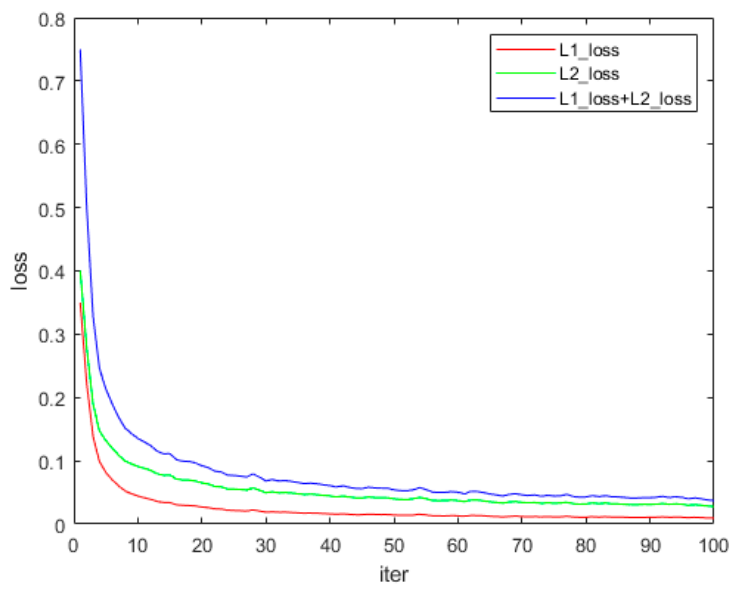

(a)

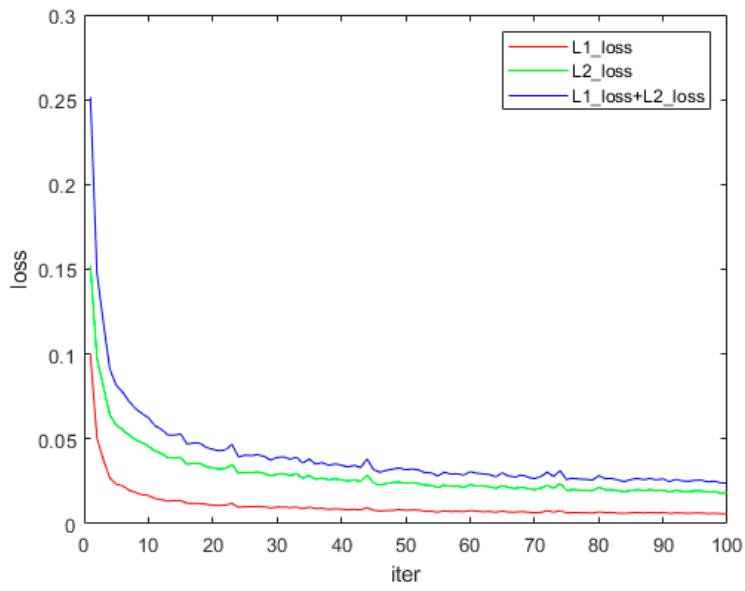

(b)

Figure 6. Rendered image training process. (a) is the training process of red laser, and (b) is the training process of green laser.

The results of laser line extraction of these rendered images are shown in Figure 7.
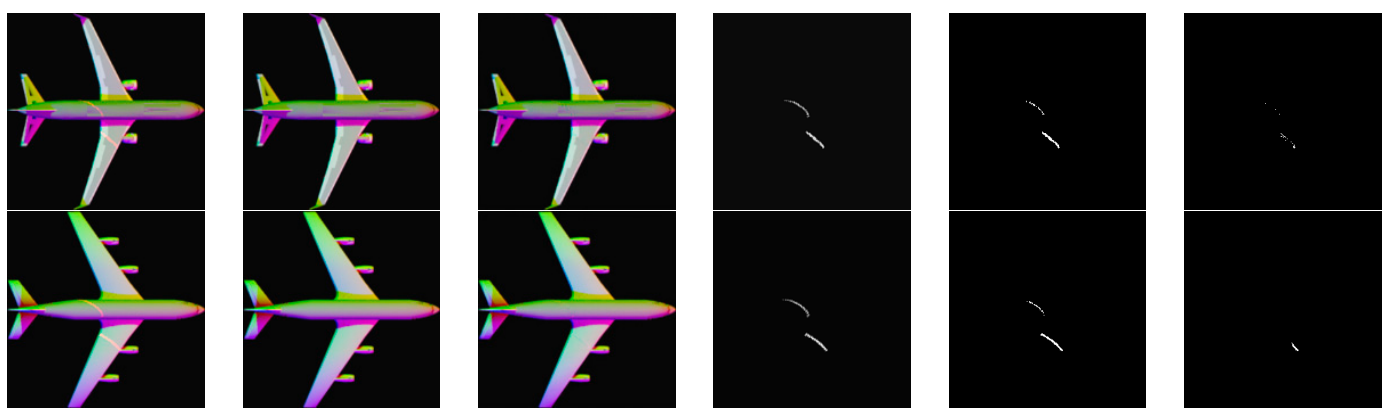

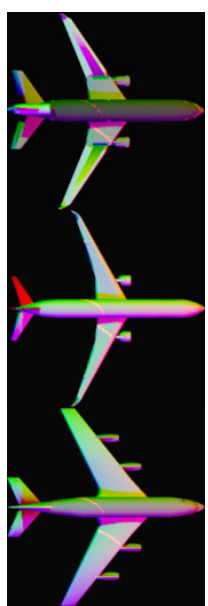

(a)

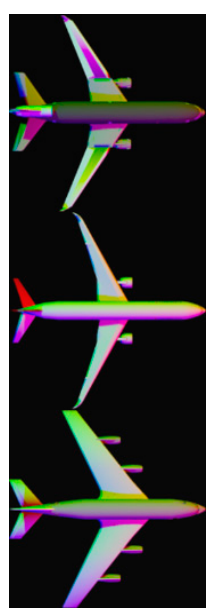

(b)

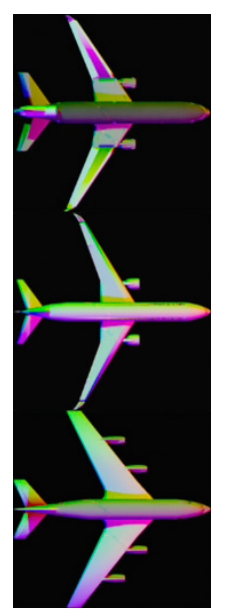

(c)

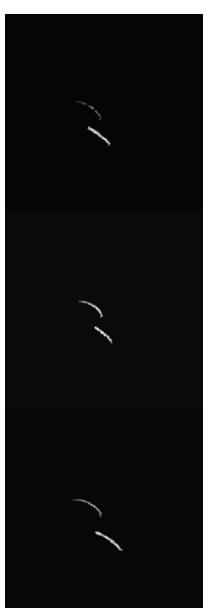

(d)

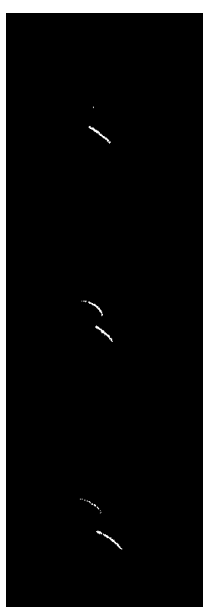

(e)

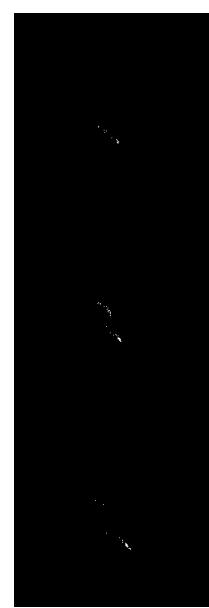

(f)

Figure 7. Processing results of rendered images. (a) are the input multispectral images with laser lines, (b) are the ground truth, (c) are the output results of our network, (d) are the ground truth of the laser images, (e) are the predicted laser images, (f) are the laser prediction error images.

\subsubsection{Extraction Results of Laser Lines in Real-World Images}

After training 100 times through the rendered image, three of the real images were input into the network as the training set. After training 500 times, the intermediate result image was obtained. Then the laser image is obtained through thresholding segmenta- 
tion, median filtering and morphological operations. After experimental debugging, the threshold was set to 0.35 in the experiment.

The real image training process is shown in Figure 8, where (a) is the shell training process and (b) is the ship model training process. The laser line extraction results using real images are shown in Figure 9.

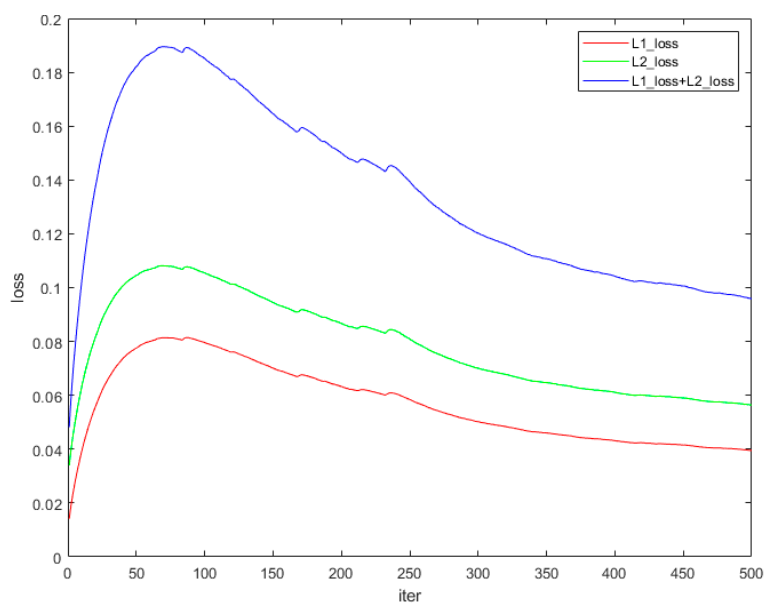

(a)

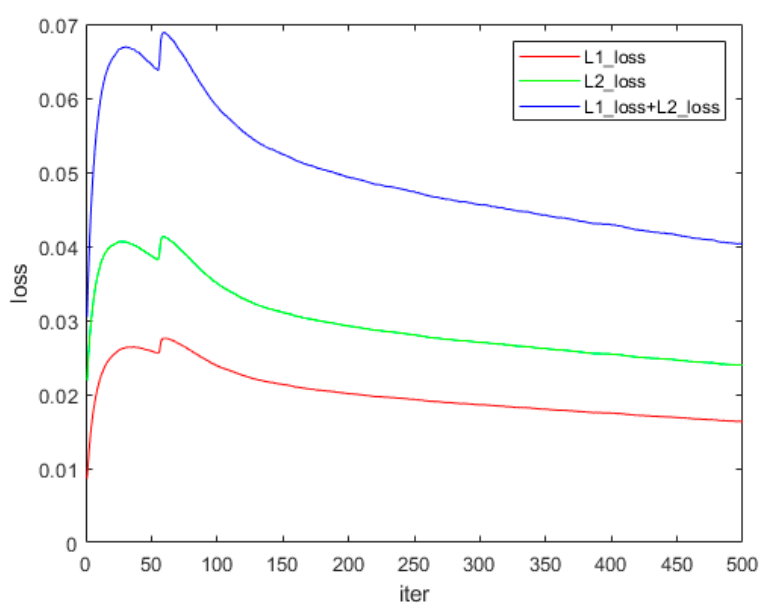

(b)

Figure 8. Processing results of real-world images. (a) is the training process of red laser, and (b) is the training process of green laser.

In Figure 8, we can see that in the initial stage of training, the loss of the network shows an upward trend, and then slowly declines. This may be because during the training of the rendered images, the network learned to find the position of the laser line autonomously, and estimate the pixel-level RGB values of the pixels covered by the laser line. After the introduction of real images, the network loss increases at the beginning of training because our network uses pixel values as input, and factors such as target's shape, albedo, and chromaticity will cause changes in pixel values. So, the network needs a small amount of training to interference items such as shape, albedo and chromaticity are eliminated, and regains the capability to find the position of the laser line. 


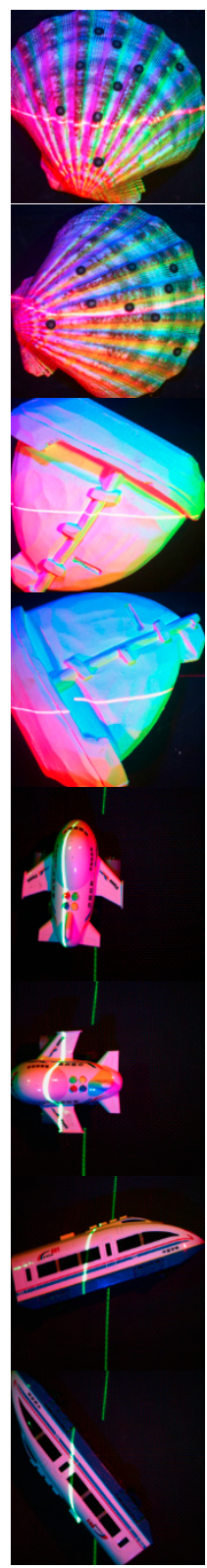

(a)

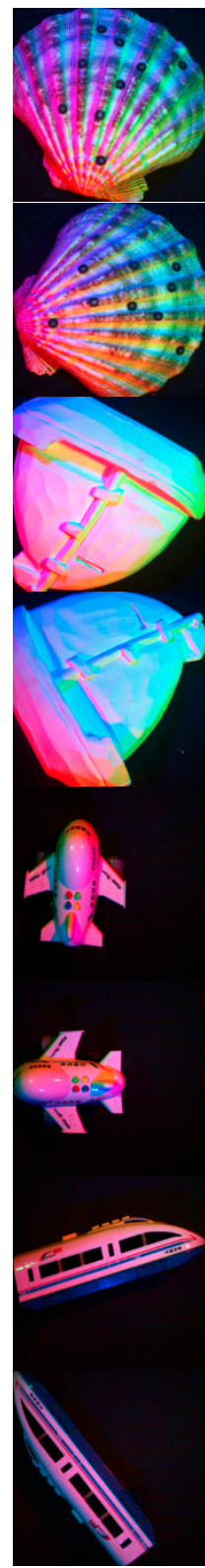

(b)

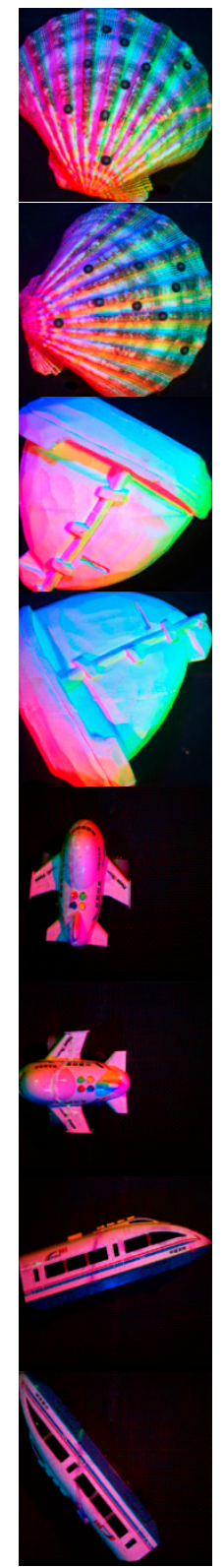

(c)
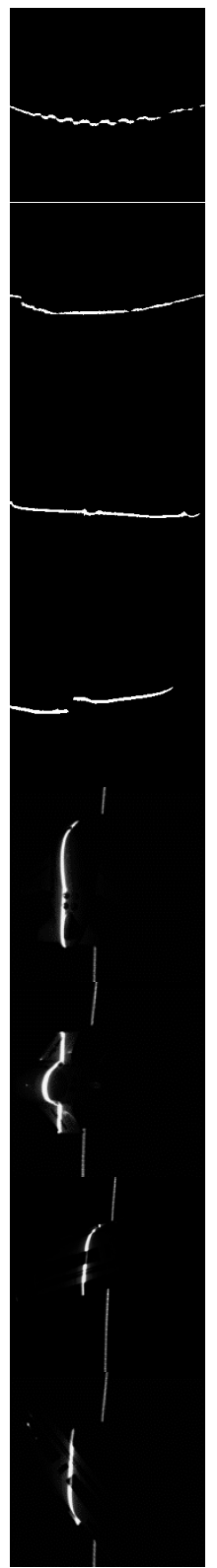

(d)

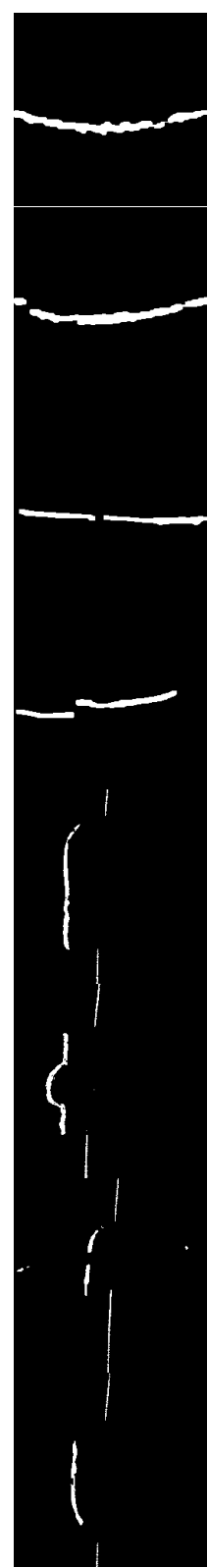

(e)

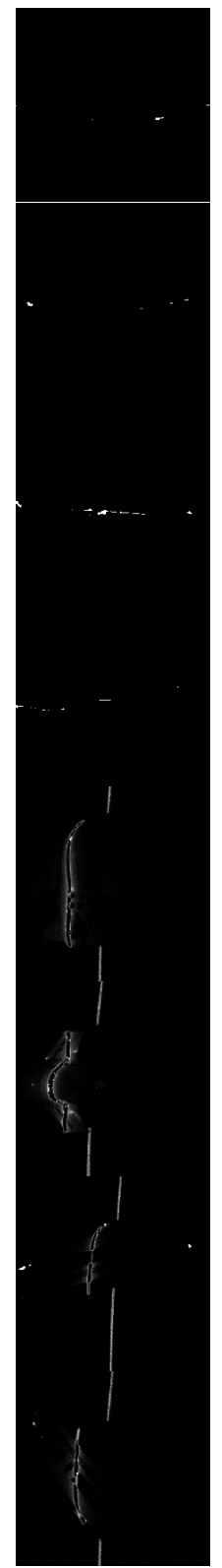

(f)

Figure 9. Real-world images processing results. (a) are the input multi-spectral images with laser lines, (b) are the ground truth, (c) are the output results of our network, (d) are the ground truth of the laser images, (e) are the predicted laser images, (f) are the laser prediction error images.

\subsection{Analysis and Discussion}

4.3.1. Comparison with the Results of Isola's Network

Comparing the results of our network with the results of the algorithm proposed by Isola [17], the algorithm we proposed can effectively suppress the pixel value in the laser line area, while the network is to suppress all the pixel values in the image, thus the desired result cannot be obtained. These results of the network are shown in Figure 10. 


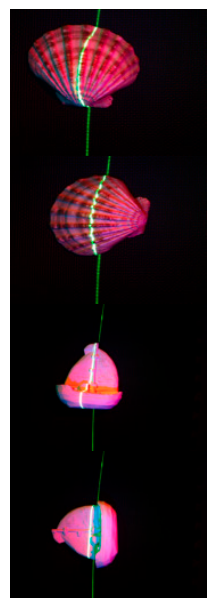

(a)

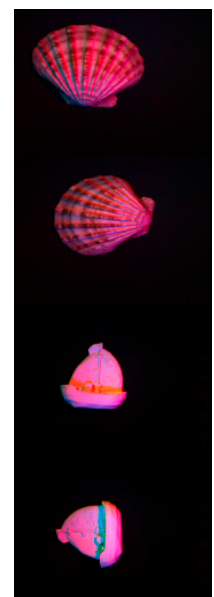

(b)

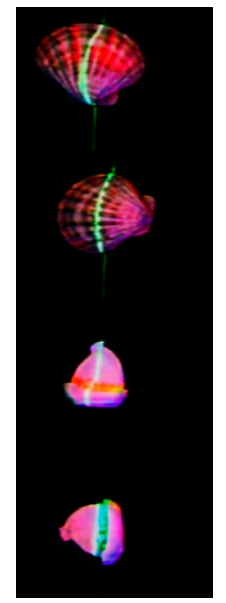

(c)

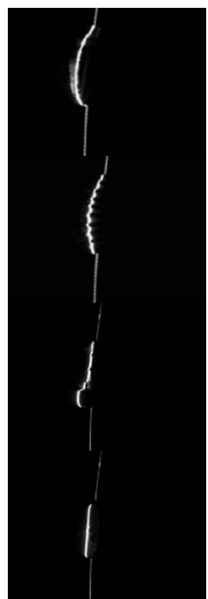

(d)

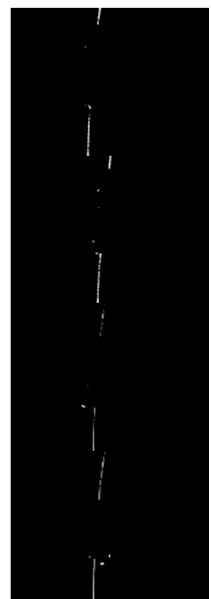

(e)

Figure 10. The result of the network proposed by Isola [17]. (a) are the input multi-spectral images with laser lines, (b) are the ground truth, (c) are the output results of the network, (d) are the ground truth of the laser images, (e) are the predicted laser images of the network.

\subsubsection{Analysis of the Added Strategy}

Based on the network proposed by Isola [17], we added three strategies, which was a U-Net-like strategy, a SqueezeNet-like strategy, and a scaling strategy of feature map. Figure 11 shows the results when we add one, two and all strategies to the network.

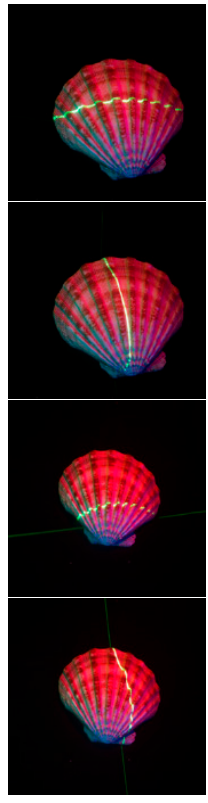

(a)

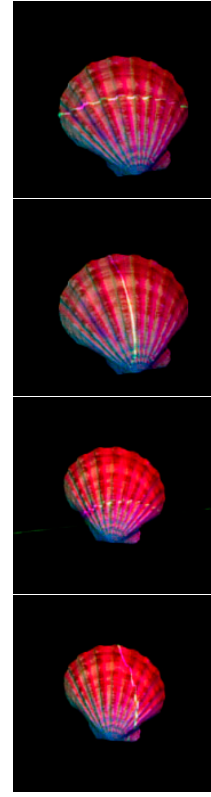

(b)

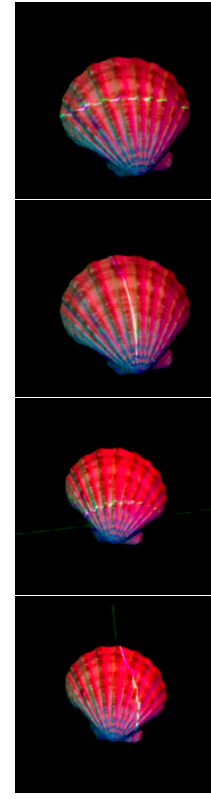

(c)

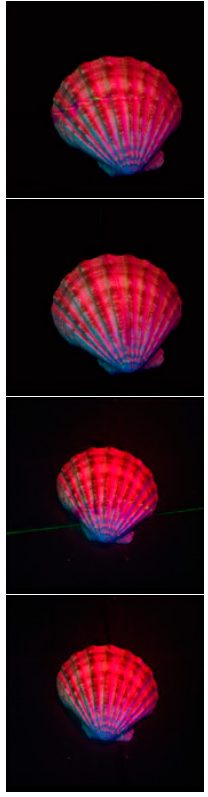

(d)

Figure 11. Analysis results of the added strategy. (a) are the input multi-spectral images with laser lines, (b) are the results when we add the scaling strategy of feature map to the network, (c) are the results when we add the scaling strategy of feature map and the SqueezeNet-like strategy to the network, and (d) are the results when we add all the three strategies to the network.

\subsubsection{Comparison with Other Image In-Painting Algorithms}

Comparing the results of our network with the results of the algorithm proposed by Criminisi $[37,38]$, we further verify the advantage of the proposed network. These comparison results are shown in Figure 12 and Table 2, where all the analysis results are compared in the laser line area obtained in the previous step. 
(1)

(2)

(3)
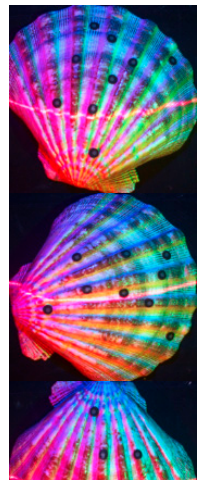

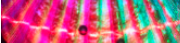

$4: 0$

0.72 ?

(4)

(5)

(6)

(7)
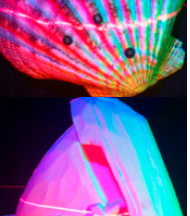

)

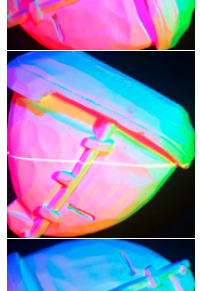

(8)

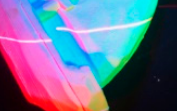

(8)

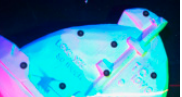

(9) 1 is

(10)

(11) $\sqrt{7}$

(12)

$\frac{\pi}{2 x}$
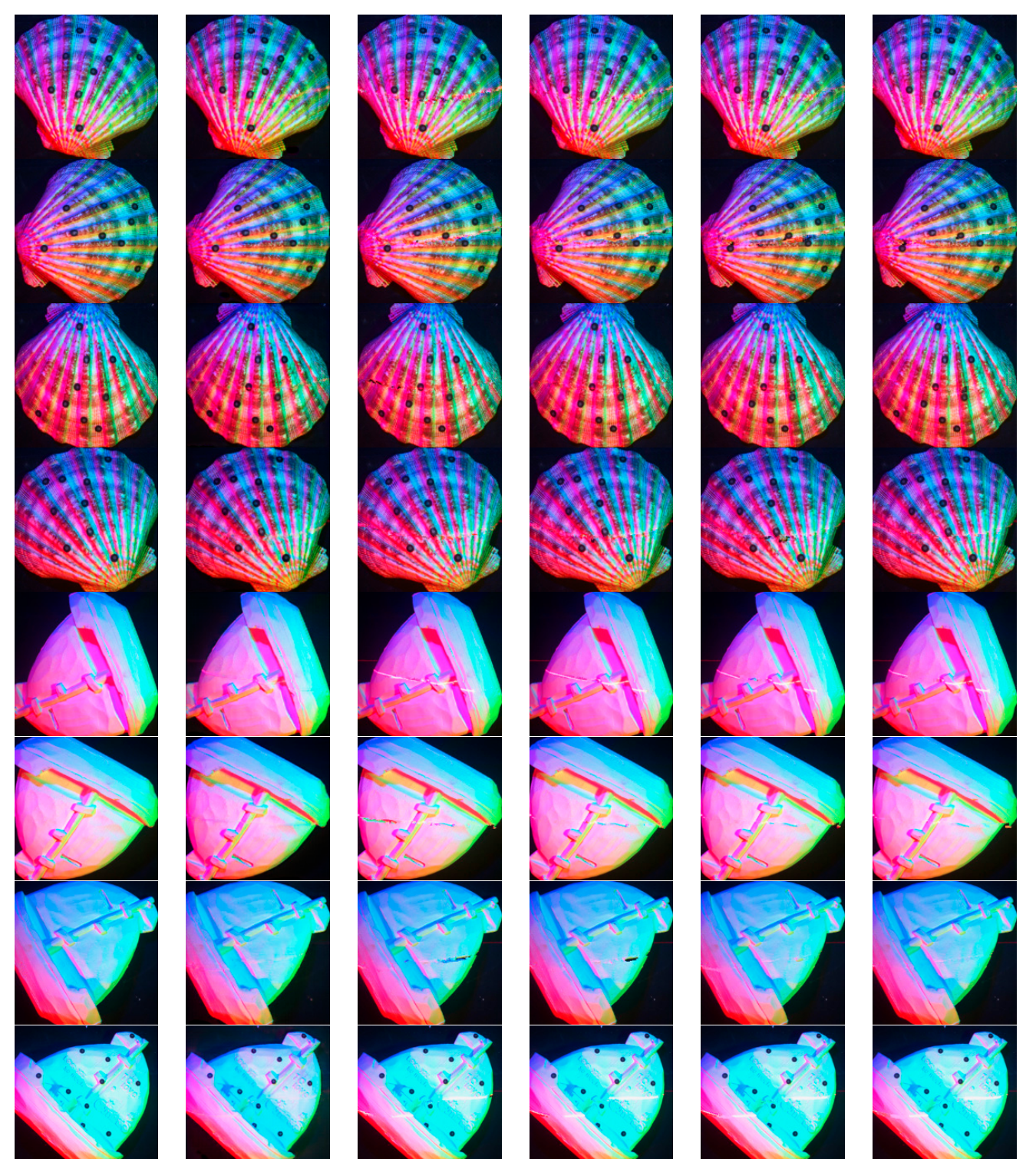

1.

18

aid
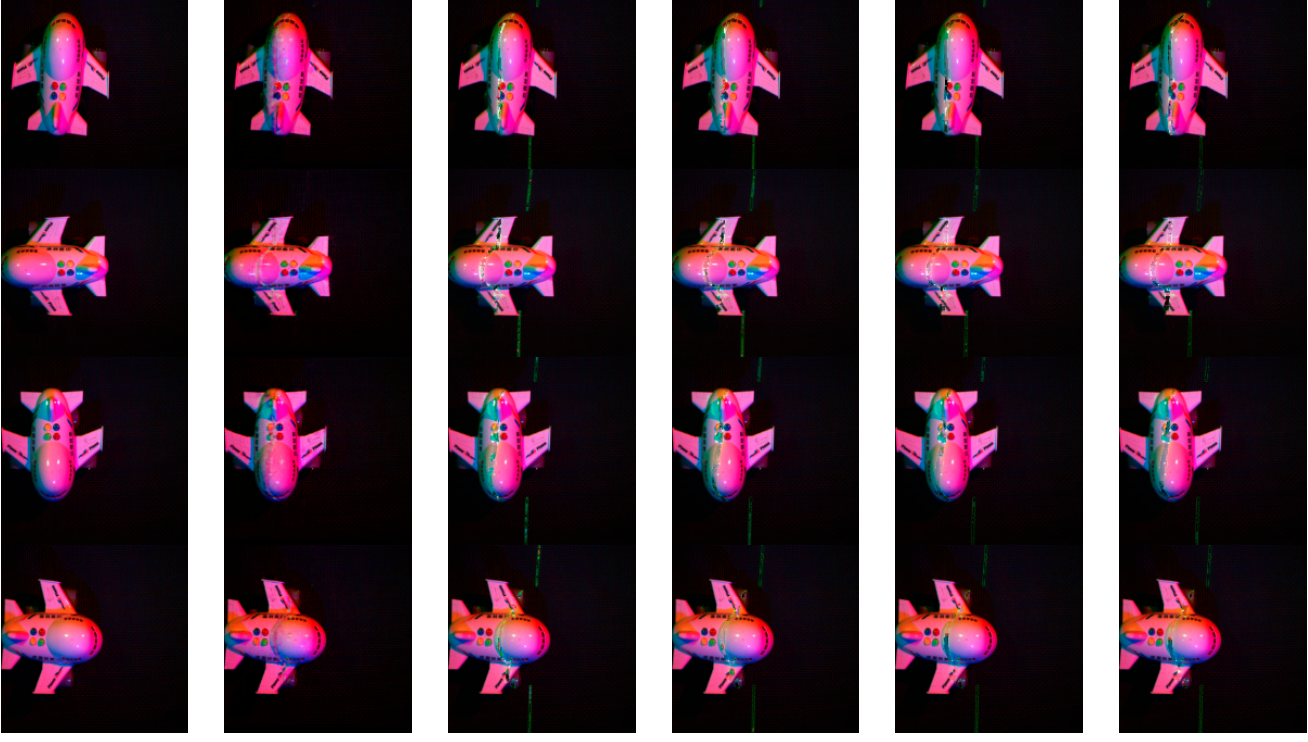

Figure 12. Cont. 
(13)

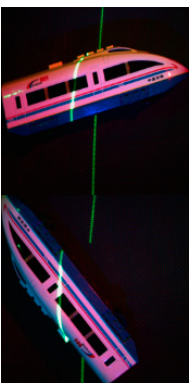

(a)

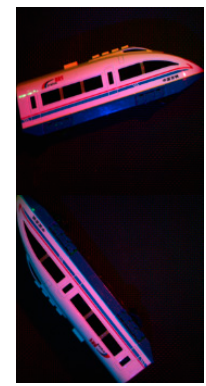

(b)

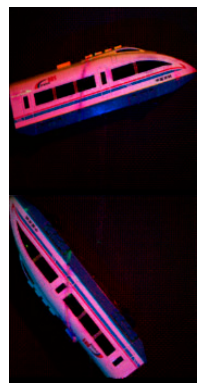

(c)

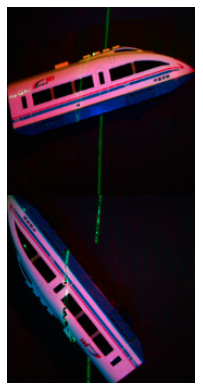

(d)

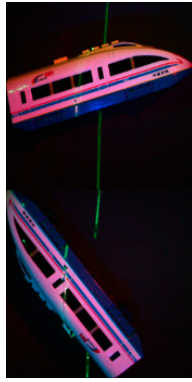

(e)

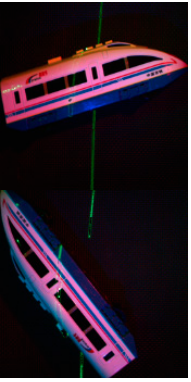

(f)

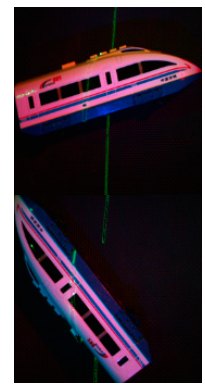

(g)

Figure 12. Compares the results of the image completion algorithm proposed by Criminisi (a) are the multi-spectral images with laser lines, (b) are the ground truth, (c) are the output results of our network, $(\mathbf{d}-\mathbf{g})$ are the results of the algorithm proposed in Criminisi's article, when the parameter "patch_size" is set to 3, 5, 7, and 9.

Table 2. Compare the results of the image completion algorithm in Criminisi's article.

\begin{tabular}{|c|c|c|c|c|c|}
\hline \multirow[t]{2}{*}{ Image } & \multirow{2}{*}{$\begin{array}{l}\text { MSE of Our } \\
\text { Result }\end{array}$} & \multicolumn{4}{|c|}{$\begin{array}{l}\text { MSE of the Result of Criminisi's Algorithm } \\
\text { When the Parameter "patch_size" Is }\end{array}$} \\
\hline & & 3 & 5 & 7 & 9 \\
\hline (1) & 0.0040 & 0.0073 & 0.0065 & 0.0060 & 0.0057 \\
\hline (2) & 0.0037 & 0.0083 & 0.0083 & 0.0094 & 0.0084 \\
\hline (3) & 0.0030 & 0.0055 & 0.0050 & 0.0052 & 0.0055 \\
\hline (4) & 0.0044 & 0.0068 & 0.0071 & 0.0068 & 0.0060 \\
\hline (5) & 0.0035 & 0.0075 & 0.0072 & 0.0066 & 0.0050 \\
\hline (6) & 0.0032 & 0.0082 & 0.0057 & 0.0053 & 0.0056 \\
\hline (7) & 0.0031 & 0.0079 & 0.0086 & 0.0037 & 0.0025 \\
\hline (8) & 0.0059 & 0.0088 & 0.0083 & 0.0078 & 0.0061 \\
\hline (9) & 0.0081 & 0.0140 & 0.0123 & 0.0132 & 0.0147 \\
\hline (10) & 0.0049 & 0.0125 & 0.0113 & 0.0091 & 0.0104 \\
\hline (11) & 0.0066 & 0.0114 & 0.0098 & 0.0096 & 0.0102 \\
\hline (12) & 0.0066 & 0.0127 & 0.0102 & 0.0098 & 0.0095 \\
\hline (13) & 0.0085 & 0.0092 & 0.0104 & 0.0094 & 0.0090 \\
\hline (14) & 0.0076 & 0.0163 & 0.0152 & 0.0131 & 0.0100 \\
\hline
\end{tabular}

Furthermore, we also compared the results of our network with those of Lu's algorithm [39] and Zeng's algorithm [30] (http:/ / 47.57.135.203:2333/, accessed on 5 January 2021), which are the newest algorithms of image in-painting.

The compared the results of our network with those of Lu's algorithm are shown in Figure 13 and Table 3.

(1)
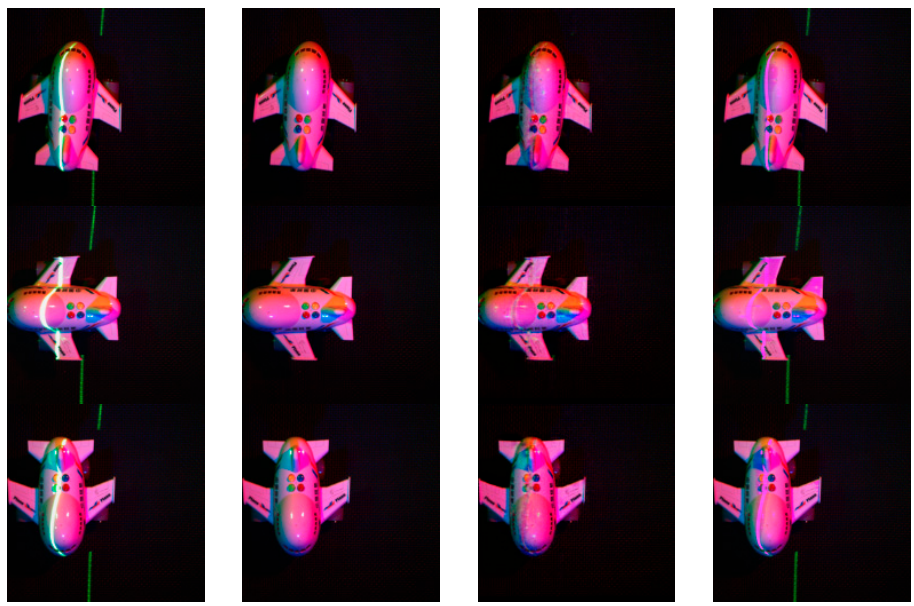

Figure 13. Cont. 
(4)

(5)

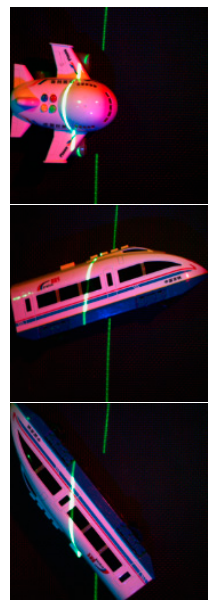

(a)

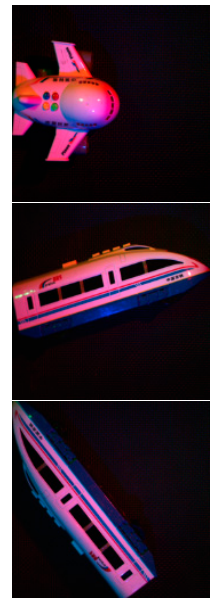

(b)

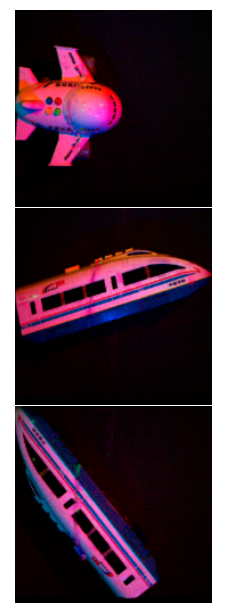

(c)

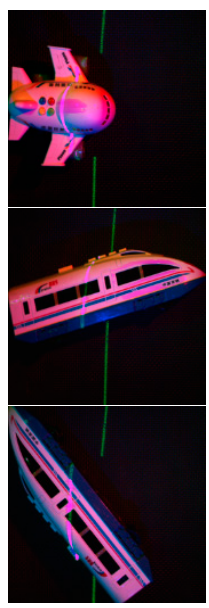

(d)

Figure 13. Compares the results of our network with Lu's algorithm. (a) are the multi-spectral images with laser lines, (b) are the ground truth, (c) are the output results of our network, and (d) are the output results of Lu's algorithm.

Table 3. Compares the results of our network with Lu's algorithm.

\begin{tabular}{ccc}
\hline Image & MSE of Our Result & $\begin{array}{c}\text { MSE of the Result of Lu's } \\
\text { Algorithm }\end{array}$ \\
\hline$(1)$ & 0.0081 & 0.0231 \\
$(2)$ & 0.0049 & 0.0267 \\
$(3)$ & 0.0066 & 0.0259 \\
$(4)$ & 0.0066 & 0.0266 \\
$(5)$ & 0.0085 & 0.0279 \\
$(6)$ & 0.0076 & 0.0307 \\
\hline
\end{tabular}

The compared the results of our network with those of Zeng's algorithm are shown in Figure 14 and Table 4.

(1)

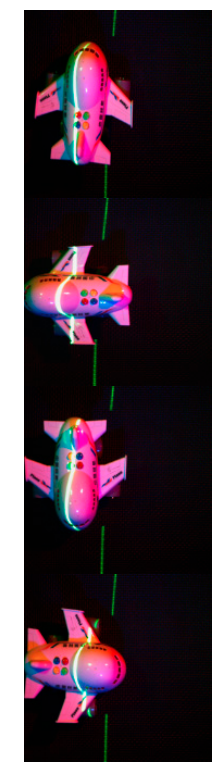

(a)

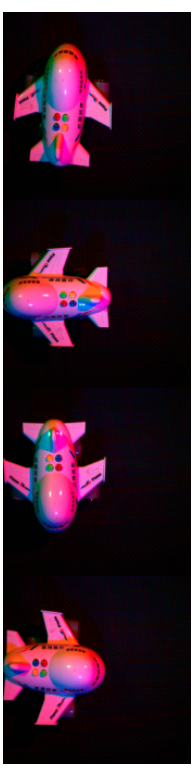

(b)

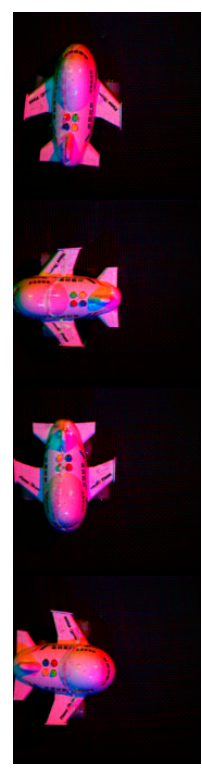

(c)

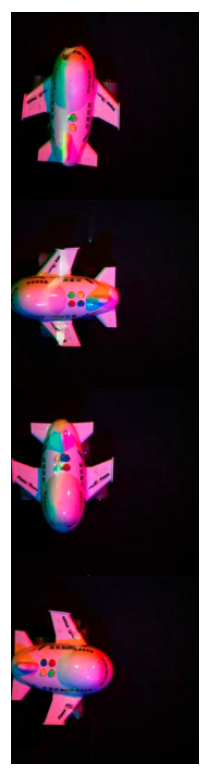

(d)

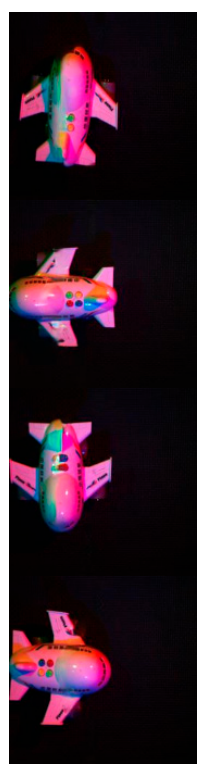

(e)

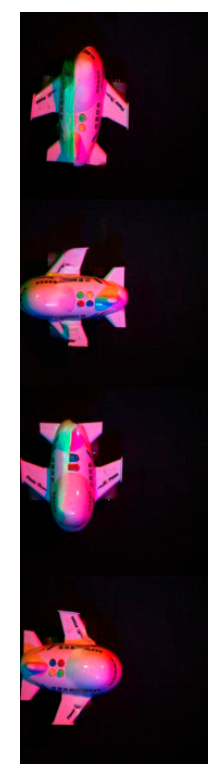

(f)

Figure 14. Compares the results of our network with Zeng's algorithm. (a) are the multi-spectral images with laser lines, (b) are the ground truth, (c) are the output results of our network, and (d-f) are the output results of Zeng's algorithm, when the parameter "Line width" is set to 9, 13 and 17. 
Table 4. Compares the results of our network with Zeng's algorithm.

\begin{tabular}{ccccc}
\hline \multirow{2}{*}{ Image } & MSE of Our & \multicolumn{3}{c}{$\begin{array}{c}\text { MSE of the Result of Zeng's Algorithm } \\
\text { When the Parameter “Line width" Is Set to }\end{array}$} \\
\cline { 3 - 4 } & & $\mathbf{9}$ & $\mathbf{1 3}$ & $\mathbf{1 7}$ \\
\hline$(1)$ & 0.0081 & 0.0141 & 0.0120 & 0.0131 \\
$(2)$ & 0.0049 & 0.0113 & 0.0079 & 0.0082 \\
$(3)$ & 0.0066 & 0.0090 & 0.0103 & 0.0113 \\
$(4)$ & 0.0066 & 0.0078 & 0.0098 & 0.0093 \\
\hline
\end{tabular}

\subsection{Reconstruction Results Using Multi-Spectral Photometric Stereo}

We input the result of our proposed network into the network of $\mathrm{Lu}$ [7] to obtain the predicted depth image and acquire the three-dimensional reconstruction result of the target using the photometric stereo algorithm based on cross laser correction Fan [16]. The result is shown in Figure 15.

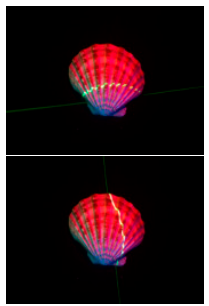

(a)

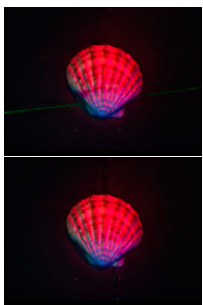

(b)

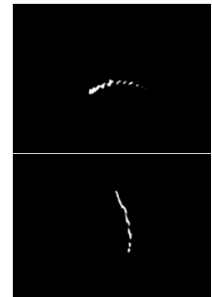

(c)

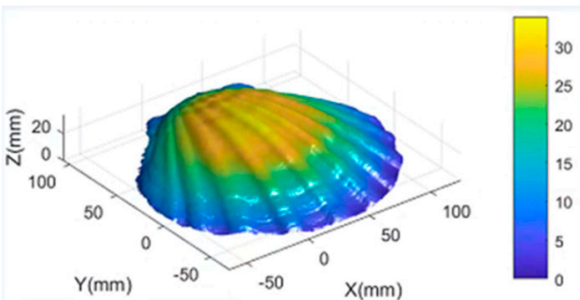

(d)

Figure 15. The three-dimensional reconstruction result. (a) are the input multi-spectral images with a laser line, (b) are the output results of our proposed network, (c) are the results of laser line extraction, and (d) is the three-dimensional reconstruction result obtained by substituting the above results into Fan's algorithm.

\section{Conclusions}

In this work, we have studied the three-dimensional reconstruction algorithm of multi-spectral images with laser lines. The Generative Adversarial Network based on image in-painting has generated laser images and multi-spectral images without laser lines, and realized the three-dimensional reconstruction based on multi-spectral images. A large number of experiments show that the proposed network can effectively extract the laser lines in the multi-spectral images with laser lines, and at the same time, the pixel values of the area covered by the laser lines are also effectively estimated.

However, there is still some room for improvement of the proposed algorithm. For example, in the process of converting the output of the network into a laser image, it is still necessary to set a threshold for morphological processing. In addition, the main application scenario of the algorithm proposed in this article is the three-dimensional reconstruction of underwater images, however, the images which this network can process effectively are acquired in the air, and the result of underwater images in-painting is not ideal. Continue to improve the network to achieve automatic laser line extraction and pixel value estimation for underwater images will be the next goal of our continued research.

Author Contributions: Conceptualization, L.L.; methodology, L.L.; software, L.L.; validation, L.L. and H.Z. (Hongbao Zhu); resources, L.L. and H.Z. (Hongbao Zhu); data curation, L.L. and Y.J.; writing—original draft preparation, L.L.; writing—review and editing, J.D. and H.Z. (Huiyu Zhou); project administration, J.D.; funding acquisition, J.D. All authors have read and agreed to the published version of the manuscript.

Funding: This research was funded by the Natural Science Foundation of Shandong Province of China (ZR2018ZB0852).

Institutional Review Board Statement: Not applicable. 
Informed Consent Statement: Not applicable.

Data Availability Statement: Not applicable.

Conflicts of Interest: Authors have no conflict of interest to declare.

\section{References}

1. Zeng, H.; Chen, Y.; Zhang, Z.; Wang, C.; Li, J. Reconstruction of 3D Zebra Crossings from Mobile Laser Scanning Point Clouds. In Proceedings of the IGARSS 2019-2019 IEEE International Geoscience and Remote Sensing Symposium, Yokohama, Japan, 28 July-2 August 2019; pp. 1899-1902.

2. Cui, Y.; Li, Q.; Yang, B.; Xiao, W.; Chen, C.; Dong, Z. Automatic 3-D Reconstruction of Indoor Environment with Mobile Laser Scanning Point Clouds. IEEE J. Sel. Top. Appl. Earth Obs. Remote Sens. 2019, 12, 3117-3130. [CrossRef]

3. Liu, T.; Wang, N.; Fu, Q.; Zhang, Y.; Wang, M. Research on 3D Reconstruction Method Based on Laser Rotation Scanning. In Proceedings of the 2019 IEEE International Conference on Mechatronics and Automation (ICMA), Tianjin, China, 4-7 August 2019; pp. 1600-1604.

4. Woodham, R.J. Photometric method for determining surface orientation from multiple images. Opt. Eng. 1980, 19, 139-191. [CrossRef]

5. Torresani, L.; Hertzmann, A.; Bregler, C. Nonrigid Structure-from-Motion: Estimating Shape and Motion with Hierarchical Priors. IEEE Trans. Pattern Anal. Mach. Intell. 2008, 30, 878-892. [CrossRef] [PubMed]

6. Chen, X.; Wu, Q.; Wang, S. Research on 3D Reconstruction Based on Multiple Views. In Proceedings of the 2018 13th International Conference on Computer Science \& Education (ICCSE), Colombo, Sri Lanka, 8-11 August 2018; pp. 1-5.

7. Lu, L.; Qi, L.; Luo, Y.; Jiao, H.; Dong, J. Three-Dimensional Reconstruction from Single Image Base on Combination of CNN and Multi-Spectral Photometric Stereo. Sensors 2018, 18, 764. [CrossRef] [PubMed]

8. Ju, Y.; Qi, L.; Zhou, H.; Dong, J.; Lu, L. Demultiplexing Colored Images for Multispectral Photometric Stereo via Deep Neural Networks. IEEE Access 2018, 6, 30804-30818. [CrossRef]

9. Ju, Y.; Dong, X.; Wang, Y.; Qi, L.; Dong, J. A dual-cue network for multispectral photometric stereo. Pattern Recognit. 2020, 100, 0031-3203. [CrossRef]

10. Ju, Y.; Qi, L.; He, J.; Dong, X.; Gao, F.; Dong, J. MPS-Net: Learning to recover surface normal for multispectral photometric stereo. Neurocomputing 2020, 375, 62-70. [CrossRef]

11. Christensen, P.H.; Shapiro, L.G. Three-dimensional shape from color photometric stereo. Int. J. Comput. Vis. 1994, 13, $213-227$. [CrossRef]

12. Ikeda, O.; Duan, Y. Color Photometric Stereo for Albedo and Shape Reconstruction. In Proceedings of the 2008 IEEE Workshop on Applications of Computer Vision, Copper Mountain, CO, USA, 7-9 January 2008; pp. 1-6.

13. Ikeda, O. Color photometric stereo for directional diffuse object. In Proceedings of the 2009 Workshop on Applications of Computer Vision (WACV), Snowbird, UT, USA, 7-8 December 2009; pp. 1-6.

14. Anderson, R.; Stenger, B.; Cipolla, R. Color photometric stereo for multicolored surfaces. In Proceedings of the 2011 International Conference on Computer Vision, Barcelona, Spain, 6-13 November 2011; pp. 2182-2189.

15. Fan, H.; Qi, L.; Wang, N.; Dong, J.; Chen, Y.; Yu, H. Deviation correction method for close-range photometric stereo with nonuniform illumination. Opt. Eng. 2017, 56, 103102. [CrossRef]

16. Fan, H.; Qi, L.; Ju, Y.; Dong, J.; Yu, H. Refractive laser triangulation and photometric stereo in underwater environment. Opt. Eng. 2017, 56, 113101. [CrossRef]

17. Isola, P.; Zhu, J.; Zhou, T.; Efros, A.A. Image-to-Image Translation with Conditional Adversarial Networks. In Proceedings of the 2017 IEEE Conference on Computer Vision and Pattern Recognition (CVPR), Honolulu, HI, USA, 21-26 July 2017; pp. 5967-5976.

18. Available online: https://www.2grobotics.com/ (accessed on 20 September 2018).

19. Mölder, A.; Martens, O.; Saar, T.; Land, R. Laser Line Detection with Sub-Pixel Accuracy. Elektron. Elektrotechnika 2014, 20 , 132-135. [CrossRef]

20. Chmelarova, N.; Chmelar, P.; Beran, L.; Rejfek, L. Improving precision of laser line detection in 3D range scanning systems. In Proceedings of the 2016 26th International Conference Radioelektronika (RADIOELEKTRONIKA), Kosice, Slovakia, 19-20 April 2016; pp. 207-212.

21. Li, Y.; Zhou, J.; Huang, F.; Liu, L. Sub-Pixel Extraction of Laser Stripe Center Using an Improved Gray-Gravity Method. Sensors 2017, 17, 814. [CrossRef]

22. Ta, H.N.; Kim, D.; Lee, S. A novel laser line detection algorithm for robot application. In Proceedings of the 2011 11th International Conference on Control, Automation and Systems, Gyeonggi-do, Korea, 26-29 October 2011; pp. 361-365.

23. Song, W.; Hu, X.; Fu, J.; Zhou, Q.; Zhou, T.; Si, P. The method of hybrid-laser image spot extracts based on HSV space SVD for power transmission line detection. In Proceedings of the 2016 IEEE International Conference on Information and Automation (ICIA), Ningbo, China, 1-3 August 2016; pp. 1361-1364.

24. Chmelar, P.; Dobrovolny, M. The Laser Line Detection for Autonomous Mapping Based on Color Segmentation. Int. J. Mech. Mater. Eng. 2013, 7, 1654-1658.

25. Liu, H.; Jiang, B.; Xiao, Y.; Yang, C. Coherent Semantic Attention for Image In-painting. In Proceedings of the International Conference on Computer Vision (ICCV 2019), Seoul, Korea, 27 October-2 November 2019; pp. 4170-4179. 
26. Hong, X.; Xiong, P.; Ji, R.; Fan, H. Deep Fusion Network for Image Completion. arXiv 2019, arXiv:1904.08060.

27. Yu, J.; Lin, Z.; Yang, J.; Shen, X.; Lu, X.; Huang, T.S. Generative Image In-painting with Contextual Attention. In Proceedings of the 2018 IEEE/CVF Conference on Computer Vision and Pattern Recognition, Salt Lake City, UT, USA, 18-23 June 2018; pp. 5505-5514. [CrossRef]

28. Yu, J.; Lin, Z.; Yang, J.; Shen, X.; Lu, X.; Huang, T. Free-Form Image In-painting with Gated Convolution. In Proceedings of the International Conference on Computer Vision (ICCV 2019), Seoul, Korea, 27 October-2 November 2019; pp. 4471-4480.

29. Nazeri, K.; Ng, E.; Joseph, T.; Qureshi, F.Z.; Ebrahimi, M. EdgeConnect: Generative Image In-painting with Adversarial Edge Learning. arXiv 2019, arXiv:1901.00212.

30. Zeng, Y.; Lin, Z.; Yang, J.; Zhang, J.; Shechtman, E.; Lu, H. High-Resolution Image In-painting with Iterative Confidence Feedback and Guided Upsampling. The 16th European Conference on Computer Vision (ECCV 2020). arXiv 2020, arXiv:2005.11742.

31. Huang, J.-B.; Kang, S.B.; Ahuja, N.; Kopf, J. Image completion using planar structure guidance. ACM Trans. Graph. 2014, 33, 1-10. [CrossRef]

32. Yang, J.; Qi, Z.; Shi, Y. Learning to Incorporate Structure Knowledge for Image In-painting. The thirty-fourth AAAI Conference on Artifieial Intelligence (AAAI 2020). arXiv 2020, arXiv:2002.04170.

33. Liu, H.; Jiang, B.; Song, Y.; Huang, W.; Yang, C. Rethinking Image In-Painting via a Mutual Encoder-Decoder with Feature Equalizations. The 16th European Conference on Computer Vision (ECCV 2020). arXiv, 2020; arXiv:2007.06929.

34. Ren, J.; Xu, L.; Yan, Q.; Sun, W. Shepard Convolutional Neural Networks. In Proceedings of the 28th International Conference on Neural Information Processing Systems_-Volume 1 (NIPS'15); MIT Press: Cambridge, MA, USA, 2015; pp. $901-909$.

35. Li, J.; Wang, N.; Zhang, L.; Du, B.; Tao, D. Recurrent Feature Reasoning for Image In-Painting. In Proceedings of the 2020 IEEE/CVF Conference on Computer Vision and Pattern Recognition (CVPR), Seattle, WA, USA, 16 November 2020; pp. $7757-7765$. [CrossRef]

36. Kingma, D.P.; Ba, J. Adam: A method for stochastic optimization. In Proceedings of the International Conference Learn, Represent (ICLR), San Diego, CA, USA, 5-8 May 2015.

37. Criminisi, A.; Pérez, P.; Toyama, K. Region Filling and Object Removal by Exemplar-Based Image Inpainting. IEEE Trans. Image Process. 2004, 13, 1200-1212. [CrossRef] [PubMed]

38. Criminisi, A.; Perez, P.; Toyama, K. Object removal by exemplar-based inpainting. In Proceedings of the 2003 IEEE Computer Society Conference on Computer Vision and Pattern Recognition, Madison, WI, USA, 18-20 June 2003.

39. Lu, C.; Feng, J.; Lin, Z.; Yan, S. Exact Low Tubal Rank Tensor Recovery from Gaussian Measurements. In Proceedings of the International Joint Conference on Artificial Intelligence (IJCAI), Stockholm, Sweden, 13-19 July 2018. 